\title{
Pseudo compartment syndrome of the calf in an athlete secondary to cystic adventitial disease of the popliteal artery
}

\section{N Ni Mhuircheartaigh, E Kavanagh, M O'Donohoe, S Eustace}

Br J Sports Med 2005;39:e36 (http://www.bjsportmed.com/cgi/content/full/39/9/e36). doi: 10.1136/bjsm.2004.015636

We report a patient with calf pain induced by sport and exercise, initially thought to represent compartment syndrome, in whom MRI and duplex ultrasound ultimately revealed cystic adventitial disease of the popliteal artery. Surgical de-roofing of the popliteal artery resulted in complete resolution of symptoms with return to sporting activities.

C alf pain is not uncommon in elite athletes occurring secondary to overuse. In most patients, pain reflects osseous insult manifest as medial tibial stress syndrome or shin splints. Less commonly, pain reflects soft tissue insult or compartment syndrome. Soft tissue insult is readily detected by clinical examination and imaging integrating ultrasound and magnetic resonance imaging (MRI). Anterolateral compartment syndrome is readily detected by pressure studies and treated by surgical fasciotomy. The diagnosis of deep posterior compartment syndrome can be more difficult, however. In this report, we present an athlete with cystic adventitial disease of the popliteal artery who presented with calf pain initially thought to be secondary to deep posterior compartment syndrome. Clinical and imaging features that allowed exclusion of compartment syndrome and ultimate diagnosis of cystic adventitial disease are discussed and the existing literature relating to this uncommon entity reviewed.

\section{CASE REPORT}

An elite athlete presented with progressive exercise induced left calf pain. In the past he had attended for partial lateral meniscectomy, but was otherwise well. At examination there was no visual or palpable abnormality and as medial tibial stress syndrome, muscle strain, or specifically deep posterior compartment syndrome was suspected, he was referred for imaging.

Review of radiographs revealed no abnormality. In an attempt to exclude compartment syndrome, MR examination of the calf was performed pre and post exercise. Post exercise images showed normal muscle signal without evidence of compartment syndrome. ${ }^{1}$ Pressure studies were not performed at this stage as T2 weighted signal in the calf muscles was normal post exercise. Entertaining the possibility of referred pain to the calf from the knee following partial meniscectomy, we performed a knee MRI. This showed no significant internal derangement, but identified a chain of cyst like structures within the wall of the popliteal vessel, producing focal compression and apparent stenosis (fig lA and B). Doppler ultrasound confirmed the presence of luminal narrowing by mural cysts (fig 1C). Subsequent gadolinium enhanced magnetic resonance angiography (MRA) of the lower limbs confirmed focal vascular narrowing (fig 1D). A diagnosis of cystic adventitial disease of the popliteal and branch arteries was made, accounting for claudicant type calf pain at presentation. The patient was referred for surgical therapy at which time de-roofing of the cysts was performed. The patient remains well at this time and has returned to sporting activities.

\section{DISCUSSION}

Calf pain in adults most frequently reflects either muscle injury, shin splints, or compartment syndrome. Vascular causes of calf pain are rare in athletes, in what is essentially a group of patients protected from the impact of atherosclerosis by age, fitness, and health. When it occurs, vascular causes of calf pain in sports persons are often overlooked and only detected late after months of morbidity.

Four causes of occlusion of the popliteal artery presenting as claudication have been previously described in sports persons: compression usually complicates an aberrant insertion of the medial head of the gastrocnemius to the intercondylar notch. ${ }^{2}$ This forces the popliteal artery to deviate medially and around the head of the muscle, which in chronicity becomes compressed. Rarely, sports persons present with claudicant type symptoms several months following direct trauma to the popliteal fossa. ${ }^{3}$ It is assumed that trauma produces local intimal damage, which in healing becomes fibrotic and focally stenosed. A similar method has been described in cyclists presenting with iliac narrowing secondary to chronic repetitive trauma, incurred by an adopted flexed position at the hips during cycling, inducing intimal fibrosis. ${ }^{4}$ Muscular hyperdevelopment in intensively trained athletes causing "functional" entrapment of the popliteal artery on exercise has also been described. ${ }^{5}$ Less commonly, as occurred in our own patient, narrowing is induced in the popliteal artery secondary to extrinsic compression by cysts developing in the vessel wall, so termed cystic adventitial disease.

Cystic adventitial disease of the popliteal artery is an uncommon condition, ${ }^{6}$ first described by Atkins and Key in $1947 . .^{6}$ It is characterised by mucin containing cysts of the adventitial tissue, causing dynamic exercise dependent flow inhibition. ${ }^{8}$ It affects males in a ratio of approximately $5: 1^{9}$ and presents predominantly in the fourth and fifth decades with symptoms of intermittent claudication. It appears to affect sporting and non-sporting individuals alike. Reflecting the demands of sport, it typically presents and becomes symptomatic earlier in sports people than in those with a more sedentary lifestyle. Reflecting age and sporting involvement, affected patients typically lack arteriosclerotic risk factors in contrast to those with peripheral arterial occlusive disease. The incidence is approximately 1 in 1200 cases of claudication or 1 in 1000 peripheral arteriograms, ${ }^{9}$ although typical diagnostic procedures like conventional angiography can be negative and therefore misleading. ${ }^{8}$ Diagnosis is often made during work up of an alternative cause of calf pain, as in our own case, where cysts were identified during an evaluation of internal structures of the knee. The intrinsic contrast afforded by MRI makes it the diagnostic test of choice. At MRI, cysts are readily identified as high signal 

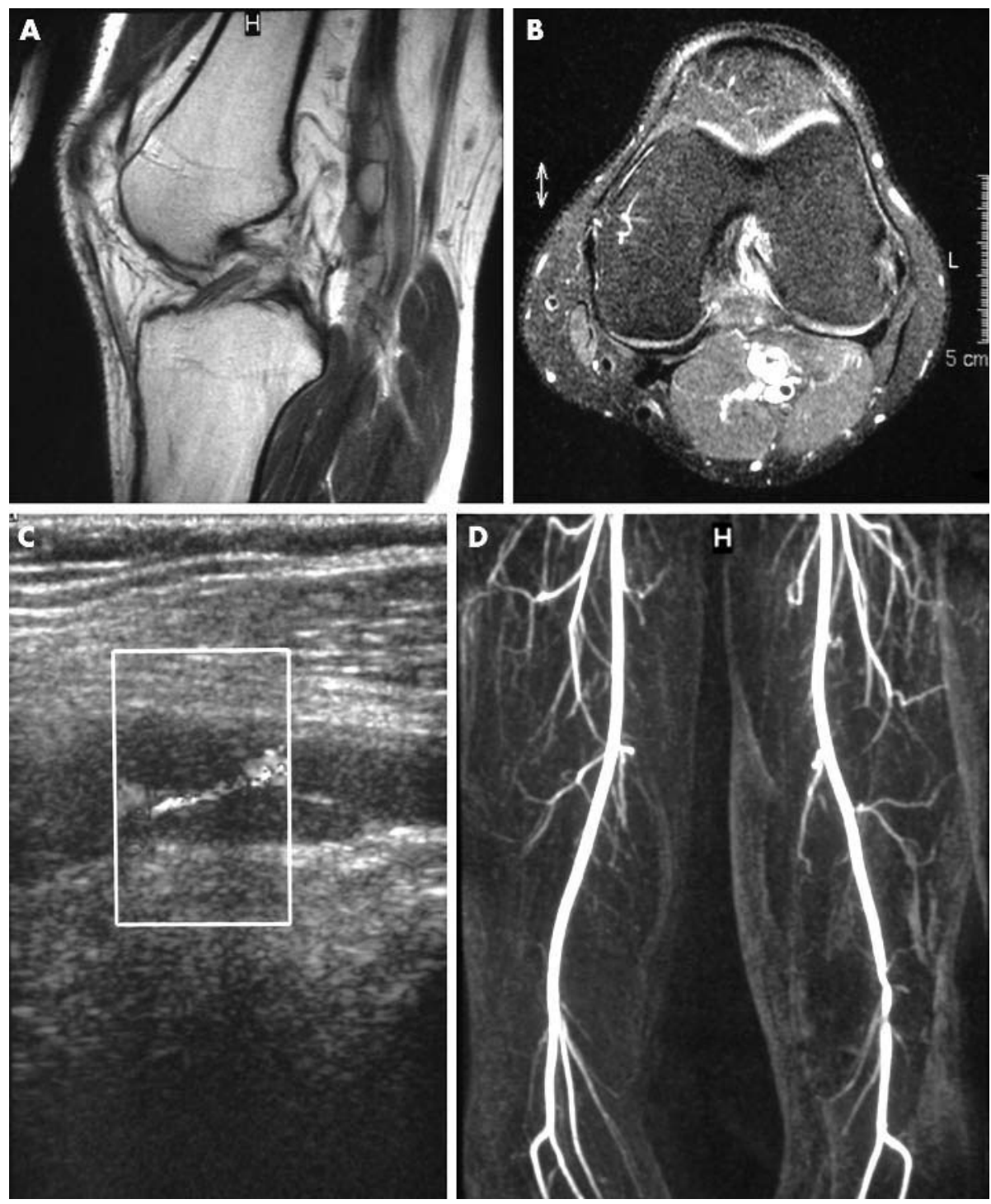

Figure 1 (A) Sagittal oblique proton density MR images of left knee showing cystic structure in popliteal fossa. (B) Axial STIR image from MR of left knee shows cysts within the wall of the popliteal artery, manifest as high signal lesions encasing popliteal flow void. (C) Doppler ultrasound examination shows an area of luminal narrowing in the popliteal artery surrounded by hypoechoic cysts within the vessel wall. (D) Maximum intensity projection contrast enhanced MRA of the lower extremities shows smooth extrinsic compression of the left popliteal artery, secondary to mural cysts.

fluid containing structures intimately related to and encasing flow void within the popliteal artery. At either conventional angiography or at gadolinium enhanced peripheral MRA, as in this case, cystic adventitial necrosis is suggested by the identification of smooth but focal luminal narrowing immediately behind the knee occurring in the presence of otherwise normal peripheral vessels. When the diagnosis is suspected, a tailored Doppler examination is helpful in differentiating intimal fibrosis from adventitial necrosis where cysts are clearly identified within the vessel wall.

Although it may be possible to aspirate cysts occurring in the vessel wall under image guidance, ${ }^{10}$ surgery is recommended in all cases to avoid the occasional dissection and thrombosis that may be induced by the image guided approach. When surgery is performed, de-roofing of the ganglion cysts may be readily undertaken. A large window is created to avoid recurrence. In severe cases where there is extensive vascular involvement, bypass jump grafts may be required to avoid the diseased vascular segment.

In summary, we present the case of an athlete presenting with progressive calf pain initially indirectly attributed to compartment syndrome, but subsequently clearly attributed to the presence of cysts within the wall of the popliteal artery, so called cystic adventitial disease.

\section{Authors' affiliations}

N N Mhuircheartaigh, E Kavanagh, M O'Donohoe, S Eustace, Mater Misericordiae Hospital, Dublin, Ireland

Competing interests: none declared

The patient detailed in this case report consented to his details being published.

Correspondence to: N Ni Mhuircheartaigh, Mater Misericordiae Hospital, Dublin, Ireland; neasa26@hotmail.com

Accepted 6 March 2005

\section{REFERENCES}

1 Verleisdonk EJ, van Gils A, van der Werken C. The diagnostic value of MRI scans for the diagnosis of chronic exertional compartment syndrome of the lower leg. Skeletal Radiol 2001;30(6):321-5. 


\section{What is already known on this topic}

Cystic adventitial disease of the popliteal artery is an uncommon condition which presents with symptoms of intermittent claudication. In contrast to peripheral vascular disease, symptoms are caused by luminal narrowing of affected blood vessels due to cysts in the adventitia. Treatment involves surgical de-roofing or, occasionally, bypass jump grafts.

2 Atilla S, Akpek ET, Yucel C, et al. MR imaging and MR angiography in popliteal artery entrapment syndrome. Eur Radiol 1998;8(6):1025-9.

3 Shute K, Rothnie NG. The etiology of cystic adventitial disease. Br I Surg 1973;60:397-400.

4 Frank R, Arko MD. Cornelius Olcott IV, MD. Arterial and venous disease in athletes. Physician Sportsmed April 2003;31(No. 4).

5 Rignault DP, Pailler JL, Lunel F. The 'functional' popliteal entrapment syndrome. Int Angiol 1985;4(3):341-3.

6 Atkins HJB, Key JA. A case of myxomatous tumour arising in the adventitia of the left external iliac artery. $B r J$ Surg 1947;34:426.

\section{What this study adds}

Cystic adventitial disease of the popliteal artery is often diagnosed during work up of an alternative cause of calf pain. This case report highlights the diagnostic modalities used to exclude some of the more common causes of calf pain encountered in athletes. Cystic adventitial disease is diagnosed by conventional angiography, MRA, or Doppler examination.

7 Brodmann M, Stark G, Pabst E, et al. Cystic adventitial degeneration of the popliteal artery - the diagnostic value of duplex sonography. Eur J Radiol 2001;38(3):209-12.

8 Tsolakis IA, Walvatne CS, Caldwell MD. Cystic adventitial disease of the popliteal artery: diagnosis and treatment. Eur J Vasc Endovasc Surg 1998;15(3):188-94.

9 Do DD, Braunschwei M, Baumgartner l, et al. Adventitial cystic disease of the popliteal artery: percutaneous US-guided aspiration. Radiology 